\title{
Proceedings of the Thoracic Society
}

The summer meeting of the Thoracic Society was held on July 12 and 13, 1963, at Gonville and Caius College, Cambridge. The meeting was composed of several symposia and some short papers. Summaries follow.

\section{Diseases of UpPer ThiRd OF THE Oesophagus}

This symposium was convened and introduced by ANDREW LOGAN.

P. R. Allison said that the only upper oesophageal webs encountered in his series appeared to be a late complication of the more general disturbance variously known as the Paterson-Brown-Kelly or Plummer-Vinson syndrome. Visualization of these webs through the oesophagoscope is reminiscent of pleural adhesions seen through the thoracoscope inasmuch as they may be so filmy as to appear and to rupture almost like a soap bubble; they may be rather tougher but dilate easily and finally rupture as the scope is passed, with very slight oozing of blood; or they may be tough and fibrous and covered with squamous epithelium. They may precede the development of carcinoma. Local treatment through the oesophagoscope or by open operation should be combined with general treatment of the anaemia and iron deficiency.

A. L. D'ABreu said that apart from Zenker's pharyngo-oesophageal diverticulum (not actually an anatomical deformity of the upper third of the oesophagus), pouches of this part of the gullet are rare; although he thought that most cysts of the mediastinum and those associated with dissociated lobes of the lung are of foregut origin, for the purpose of the discussion only duplications of the oesophagus affecting this region were mentioned. Appropriate examples of this condition were presented briefly.

The main part of the presentation concerned 37 examples of Zenker's diverticulum. Two of these were treated by Dolman's endoscopic method, two by dilatation, and one died before treatment could be offered. Thirty-two were treated by one-stage excision of the diverticulum without mortality. Results, complications, and associated, possibly aetiological, lesions were presented.

A. JACOBS said that 55 cases of dysphagia localized in the post-cricoid region had been investigated at the Cardiff Royal Infirmary in the past year. The radiological and histological appearances of the postcricoid lesion had been studied.

Haematological examination showed that the syndrome is associated not only with iron deficiency but with a severe degree of gastric atrophy giving rise to subnormal absorption of vitamin $\mathbf{B}_{12}$ in over half the cases. Three cases of pernicious anaemia were discovered.

Paterson-Kelly syndrome is closely linked with the mucosal changes which occur in the state of 'prepernicious anaemia'.
M. Lederman said that for the purposes of discussion the term upper third of the oesophagus was used so as to include tumours arising in relation to the oesophageal mouth as well as the cervical and supra-aortic portions of the mediastinal oesophagus.

The main features of the natural history of the tumours affecting these different regions of the upper third of the oesophagus were discussed and their influence on treatment shown.

Radiotherapy gives admittedly poor results except in the rare early cases: the palliative value of radiotherapy justifies its regular use. The possible combinations of radiotherapy and surgery were discussed and their limitations emphasized.

\section{World Distribution of Hydatid Disease}

L. R. WEST considered that a true picture of the extent of human hydatid disease throughout the world is difficult to obtain. The disease is notifiable in very few countries outside of South America and Australasia. Experience in New Zealand suggests that notifications considerably underestimate the true prevalence of the disease in a community.

While the sheep-farming countries, Uruguay, Argentina, and New Zealand, have immense animal reservoirs of infection and a considerable human problem, prophylactic measures are beginning to yield results. In the Mediterranean littoral, Spain, Algeria, Turkey, and Greece have serious human infection. In north-west Europe, however, the disease is now a rarity due to controlled slaughtering of livestock and improved living standards in agricultural communities.

\section{The Casoni Reaction and Hydatid Complement Fixation TeST}

C. M. P. Bradstreet analysed the results of skin tests and antibody titres obtained by the method of complement fixation in cases in which the diagnosis had subsequently been confirmed by operation. The merits of the two tests, as they are performed in this country, were considered.

The skin test, as it is performed in this country, is less reliable than the complement fixation test.

\section{Post-OPERATIVe ARrhythmias}

H. A. Fleming considered that post-operative arrhythmias are particularly common with cardiac surgery, but may follow any thoracic surgery, or indeed any general surgery in susceptible groups. He suggested that all candidates for cardiac surgery, and 
those judged likely to have arrhythmia in the course of other forms of surgery, should be digitalized preoperatively. Digitalis is an admirable drug, but the therapeutic level is frequently very close to the toxic level and its difficulties must be carefully understood. Drugs of the adrenergic beta receptor antagonist group may separate the therapeutic and the toxic effects. Whenever possible pre-operative digitalization should be carried out slowly over a period of days. If this is not possible it may be wiser to withhold digitalis and treat any developing arrhythmia by rapid digitalization. There is now much evidence that only small amounts of digitalis are lost from the body during cardiopulmonary bypass.

The differentiation of the types of post-operative arrhythmia is very important and often difficult. It is of particular importance in deciding whether the rhythm should be treated by more or by less digitalis. Analysis of a personal series of atrial septal defects was used to illustrate the incidence of these arrhythmias.

Conversion to sinus rhythm was discussed and reference made to Lown's direct current counter shock. If the early success of this method of terminating all forms of arrhythmia is confirmed, the accurate differentiation of the various types will become less important.

\section{Lung Diseases in Animals}

This symposium was introduced by L. E. HugHes, who said that, although nematodes, fungi, bacteria, mycoplasma, and viruses cause disease of the lungs in man and other mammals, little is known about the public health significance of the organisms associated with lung disease of domesticated mammals. Their host-specificity has not been well defined, and lack of spread from one species may be due to lack of opportunity rather than lack of pathogenicity. Attention was drawn to some circumstances in which respiratory diseases have spread from domesticated livestock to man.

W. H. PARKer considered that diseases of the lungs are far more common in young stock than in adults. In grazing stock parasitic bronchitis due to the presence of Dictyocaulus viviparus in the lungs is the major problem and has been the subject of a vast amount of recent research concerned with epidemiology, treatment, and vaccination. In housed calves epidemics of lung infections were already increasing in importance when the development of more intensive beef production accelerated the process in the last two to three years. Control of this problem is largely a matter of housing and husbandry, while research into the cause is directed towards mycoplasma and viruses.

A. J. Stevens described three common clinical syndromes of lung disease in sheep.

Parasitic pneumonia associated mainly with Muellerius capillaris, Cystocaulus ocreatus, and Dictyocaulus filaria is widespread affecting over $90 \%$ of sheep, but only occasionally causes serious clinical loss.

Enzootic pneumonia is an acute killing disease constantly associated with adverse managemental factors. Though Pasteurella haemolytica can be consistently isolated from pneumonic lung, there are reasons to believe that other agents, including P.P.L.O.s and viruses, are also involved.

Pulmonary adenomatosis (Jaagsiekte) is believed to be widespread, particularly in Scotland, but only occasionally causes serious loss in any one flock. Though demonstrably transmissible, the incubation (or development) period is very long, probably several years sometimes. A P.P.L.O. may again be involved, but undoubtedly managemental factors influence the incidence of the disease.

M. C. LANCASTER said that in experimental respiratory infections, both the route of administration and the infective agent directly influence the resulting lesion. Using aerosols, the large particles are filtered out in the upper respiratory tract while small particles can pass directly into the alveoli and establish infection, with discrete focal lesions, in the alveolar wall. With suspensions, a spreading bronchopneumonia distribution occurs. Viruses such as influenza result in the infection of the bronchial epithelium and the alveolar wall.

The importance of mixed viral and bacterial infections has been seen in the outbreak of staphylococcal pneumonia following infection with Asian influenza in man.

\section{A Case for Discussion}

This was presented by J. S. DAvidson, and members of the Panel were J. Leigh Collis, P. Hugh-Jones, D. M. Pryce, and G. Simon.

J. S. Davidson said that the patient, a male aged 67 at the time of his death, had a history of recurrent peptic ulceration, having had two partial gastrectomies, closure of a gastro-colic fistula, and finally a total gastrectomy (with Roux jejunal loop). Four and a half years after total gastrectomy he developed dysphagia due to ulceration and stricture of the oesophagus at the level of the aortic arch. In the last months of his life he developed diarrhoea and diabetes.

At autopsy he was found to have an islet cell carcinoma of the pancreas with metastases in the liver. The oesophagus, below the level of the ulcer, was lined by intact columnar epithelium resembling flattened small intestinal mucosa.

The case was presumed to be one of ZollingerEllison syndrome in which ulceration of the oesophagus occurred after total gastrectomy. The most interesting feature was the possible role of the oesophageal columnar epithelium under the influence of the ulcerogenic tumour in the aetiology of the ulcer.

\section{What Goes ON After Operation?}

George H. A. Clowes, Jr., dealt in detail with the changes in ventilation, alterations in acid-base equi- 
librium, and blood volume, and made some practical suggestions as to the necessity for controlled ventilation of the patient, and the overall insurance of an adequate cardiac output.

\section{Histiocytosis X}

Introduced by J. Livingstone, J. G. Lewis considered that histiocytosis $\mathrm{X}$ (esosinophilic granuloma) in adults may affect the lungs as the sole or predominant manifestation. Early histological features show discrete granulomata containing histiocytes and eosinophils, and later interstitial fibrosis, cysts, and localized emphysema. Asymptomatic forms are discovered by routine radiography; in others cough, breathlessness, and weight loss are present. Abnormal signs in the lungs are unusual until the illness is advanced; at least one quarter will have a spontaneous pneumothorax.

The typical radiographic picture is one of diffuse mottling or nodularity progressing to honeycombing.

Complete resolution can occur spontaneously or following steroid therapy. The prognosis is variable; pulmonary involvement is responsible for much of the disability and the occasional fatality in histiocytosis $\mathrm{X}$.

\section{Metastatic Chorionepithelioma}

E. N. Moyes described four cases of metastatic chorionepithelioma in young men. The primary tumour was testicular in two, in a retroperitoneal teratoma in another, and the fourth probably derived from a bladder growth. All had evidence of lung metastases and a high excretion rate of chorionic gonadotrophin.

Treatment with the folic acid antagonist, methotrexate, in combination either with chlorambucil and actinomycin D or with 6-mercaptopurine, resulted in partial remission in two cases, for 3 and 7 months respectively, with increased well-being, a decrease in the lung metastases, and a fall in the urinary output of chorionic gonadotrophin. The other two patients failed to respond.

\section{INTRATHORACIC MALIGNANT LYMPHOMA}

Introduced by $H$. M. Foreman, G. M. KIRK explained that the term malignant lymphoma includes lymphosarcoma, reticulosarcoma, Hodgkin's disease, Hodgkin's sarcoma, and follicular lymphoma.

These tumours of the reticular system involve the chest at some stage in $50 \%$ of such patients. Most commonly the mediastinal glands are affected alone. Displacement of mediastinal structures is rare, but compression may cause superior vena caval obstruction, nerve palsies, or lung collapse.

The lung may be involved along with mediastinal lymph gland enlargement and much less commonly alone. These parenchymal lesions may be single or multiple or resemble lymphangitis carcinomatosis.

Cardio-pericardial involvement may cause arrhythmias and intractable congestive cardiac failure or pericardial effusion.
Skeletal lesions may be osteolytic, osteoblastic, or mixed.

Clinically and radiologically it is difficult to distinguish the malignant lymphoma with intrathoracic lesions from other chest malignancies. Exact diagnosis is possible only by histological examination.

\section{DEEP DIVING}

This symposium was introduced by S. MiLES, who said that when man goes under water his tissues are unaffected by the changes in pressure, acting as if they were fluid within a fluid, and even at the greatest depth there is no conscious awareness of external pressure, and bodily functions continue normally.

On the contrary, the air or breathing mixture which the lungs contain is subject to Boyle's law, and if lung volume is to be maintained within normal respiratory limits it is essential to ensure that the intrapulmonary pressure is maintained at that of the surrounding water. This increased intra-alveolar pressure results in a completely new situation as far as gaseous interchange with the blood and tissues is concerned.

The whole of diving physiology is built round this fundamental consideration, and thus a knowledge of respiratory function and an appreciation of the effects of increased partial pressures of oxygen, nitrogen, and other gases are of paramount importance. The simplest approach is to consider changes in pressure, volume, and density, the reaction to increased partial pressures of oxygen and nitrogen or other gases used and, finally, responses to decrease in pressure as the diver ascends.

H. V. Hempleman considered that recent animal experiments involving breathing oxy-helium, oxynitrogen, and oxy-argon gas mixtures at pressures greater than atmospheric tend to support the view that decompression sickness is caused by the presence in the body of a fixed excess volume of gas. This excess volume may be acquired either by a long time at a comparatively low pressure or a short time at a much higher presure. The pressure-time relations thus established correspond closely with the volume-time relations obtained when a subject denitrogenates by breathing oxygen.

D. E. MACKAY said that the practical problems of deep diving can be divided into those dealing with the diver and those dealing with all his equipment. For a solution it is important that the depth, duration, purpose, and location of the dive must be decided. The technical problems will involve seamanship; control of descent and ascent; the partial pressures of each gas and the total density of the mixture breathed by the diver; the type of breathing equipment used; and communication and expense. The human problems concern selection; training; temperature regulation; reliability; response to decompression routines; availability of treatment facilities; as well as questions on diet, frequency of diving, morale, and off-duty behaviour. 